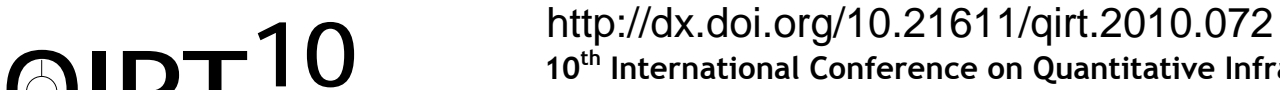 \\ $10^{\text {th }}$ International Conference on Quantitative InfraRed Thermography \\ July 27-30, 2010, Québec (Canada)
}

\section{Contrast comparison between frequency- and time-domain photoacoustic imaging}

\author{
by B. Lashkari*, A. Mandelis* \\ * Center for Advanced Diffusion-Wave Technologies (CADUFT), Mechanical \& Industrial Engineering, University \\ Toronto, 5 King's College Road, Toronto, ON M5S 3G8, Canada, Tel: 416-9781287 Fax: 416-9786160, email:
} bahman@ mie.utoronto.ca

\begin{abstract}
Photoacoustics (PA) is a promising technology for medical imaging due to its superior contrast and resolution. This technique can be implemented either in time domain by powerful nanosecond laser pulses or in frequency domain (FD) by low power intensity-modulated continuous wave (CW) chirped laser irradiation ("the photoacoustic radar").

In our previous work [1,2] FD-PA imaging with linear frequency-swept optical modulation was implemented and the application of various signal processing methods to obtain depth-selective imaging was demonstrated. Later on we compared the FD and the pulsed techniques with respect to the maximum detectable depth using a dual-mode PA system [3]. Comparable depths were found with the FD technique exhibiting significantly superior signal-to-noise ratio (SNR). Very recently [4] we demonstrated that by combining the additional imaging channel that CW method possess due to its phase information with the cross-correlation amplitude results, the contrast and resolution of the image can be improved.

In this study the contrast of images produced with both techniques is compared for various depths of a scattering medium. Experiments were implemented using two ultrasonic transducers with different center frequencies of 3.5 and 0.5 MHz. The samples consisted of two black rubber squares of areas $2 \times 2 \mathrm{~mm}^{2}$ and $4 \times 4 \mathrm{~mm}^{2}$ located at the same depth. These specifications were devised to enhance the comparison to the limits of the system, in other words, as we are trying to determine how small and how deep we can detect an absorber in a highly scattering medium, by comparing the contrast of the two rival methods. The wavelength of both pulsed and CW lasers utilized was $1064 \mathrm{~nm}$ and laser energies were controlled under the safety standard limits [5].

Since the CW method provides the capability to control the waveform shape and its bandwidth (BW), it is essential to examine the optimal BW of the frequency-modulated chirps to maximize the signal-to-noise ratio (SNR). It is demonstrated theoretically and experimentally that the optimal frequency BW does not depend exclusively on the transfer function of the ultrasonic transducer and its $-6 \mathrm{~dB}$ BW, but also on the acoustic and optical properties of the tissue and lesion. To be specific, other than the transducer's BW, acoustic attenuation of the medium and optical absorption and speed of sound in the lesion are key factors.

Some results are demonstrated in figures 1 and 2 by utilizing the high frequency transducer. Figure 1.a shows the cross-correlation amplitude in the PA radar method generated in the middle of a black rubber square of $4 \times 4 \mathrm{~mm}^{2}$ in $19 \mathrm{~mm}$ depth of a $0.47 \%$ intralipid solution. Figure $1 . b$ shows the transient response of the same sample to a pulsed laser. Comparison of these signal traces, demonstrates the higher SNR achieved by the CW method.

Figures 2.a and $b$ are the complete images produced at a specific depth corresponding to the surface of the black rubber samples. These images are constructed by complete scanning of a $15 \times 15 \mathrm{~mm}^{2}$ area using the CW and pulsed methods. It demonstrates that at the ultimate depth for the high frequency transducer, the pulsed laser achieved better detectivity. Nevertheless by employing the additional phase channel it is demonstrated that the contrast and resolution of the CW method is superior for shallower absorbers in the case of high frequency transducer.

Next experiment exhibits the results of imaging a sample (absorption coefficient: $2 \mathrm{~cm}^{-1}$ ) in different depths from 18 to $26 \mathrm{~mm}$ of $0.47 \%$ Intralipid solution using the low frequency transducer. Figures $3 . a$ is the images generated by the amplitude of the CW method filtered by its phase channel. Figures $3 . b$ is the images generated by the pulsed method. These images demonstrate comparable detectivity for both methods using the low frequency transducer and superior contrast of the CW method regardless of the absorber's depth.
\end{abstract}



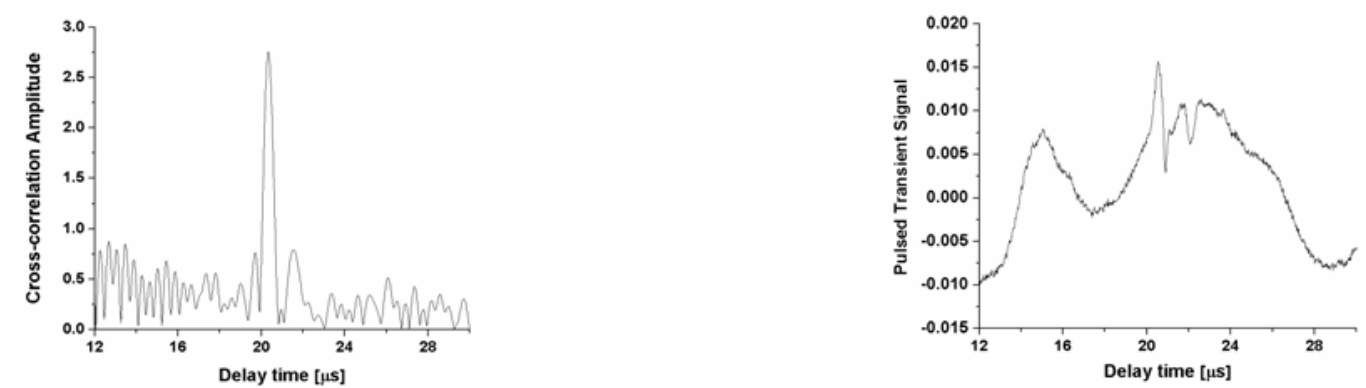

Fig. 1. a. Cross-correlation amplitude signal generated by FD PA. b. Signal trace generated by pulsed laser PA.

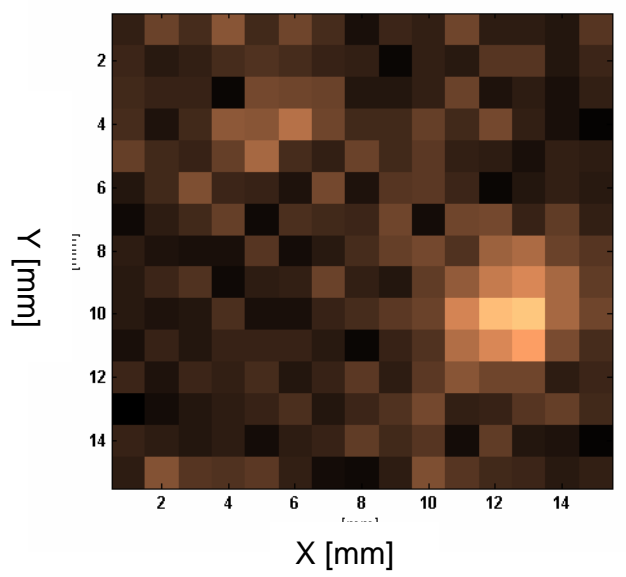

Fig. 2. a. Image generated by FD-PA.

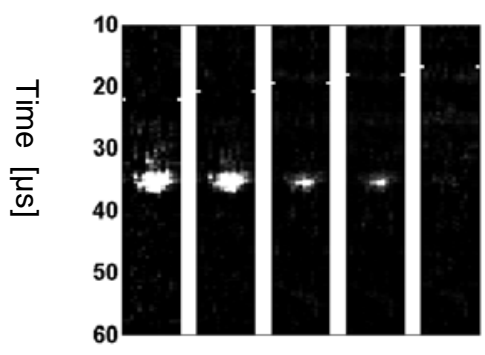

Fig. 3. a. Images generated by FD-PA.

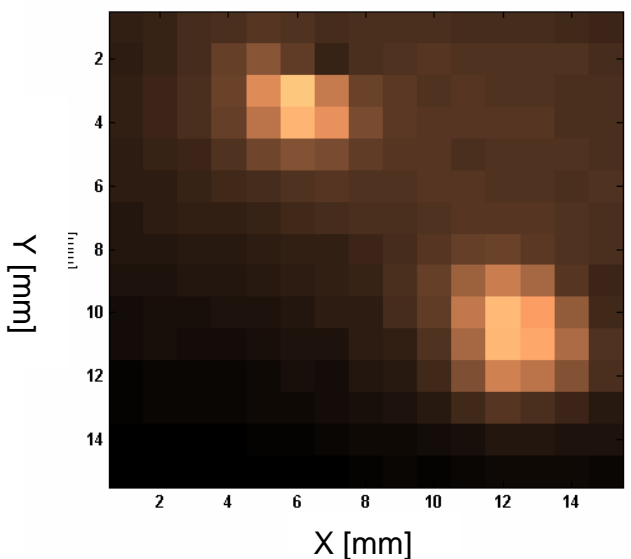

b. Image generated by pulsed laser PA.

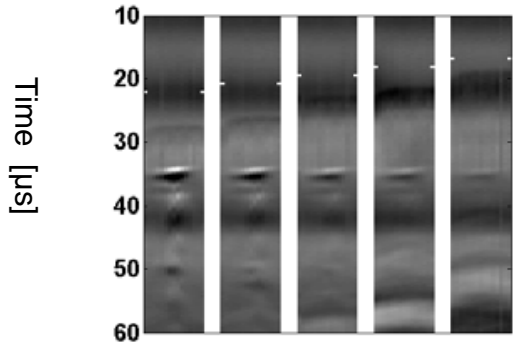

b. Images generated by pulsed laser PA

\section{REFERENCES}

[1] S.A. Telenkov, and A. Mandelis, "Fourier-domain biophotoacoustic subsurface depth selective amplitude and phase imaging of turbid phantoms and biological tissue,"J. Biomed. Opt. 11, 044006, (2006).

[2] S.A. Telenkov, and A. Mandelis, B. Lashkari, and M. Forcht, "Frequency-domain photothermoacoustics: Alternative imaging modality of biological tissues," J. Appl. Phys. 105, 102029, (2009).

[3] S.A. Telenkov, and A. Mandelis, "P hotothermoacoustic imaging of biological tissues: maximum depth characterization comparison of time and frequency-domain measurements,"J . Biomed. Opt. 14(4), 044025, (2009).

[4] B. Lashkari, and A. Mandelis, "Signal-to-Noise Ratio, Contrast and Resolution Enhancement in Frequency-Domain Photothermoacoustic Imaging Using Nonlinear Chirp Modulation", To be published in Optics Letters.

[5] American National Standards Institute, American National Standard for the Safe Use of Lasers in Health Care Facilities: Standard Z136.1-1993. 